\title{
FIXED POINT THEOREM FOR A PAIR OF SELF MAPS SATISFYING A GENERAL CONTRACTIVE CONDITION OF INTEGRAL TYPE
}

\author{
${ }^{1}$ M. R. Singh*, ${ }^{2}$ L. Sharmeswar Singh \\ ${ }^{1}$ Dept. of Mathematics, Manipur University, Canchipur. Imphal-795003(Manipur), India. \\ ${ }^{2}$ Dept. of Mathematics, D. M. College of Teacher Education Imphal-795001(Manipur), India. \\ *Corresponding author: mranjitmu@ rediffmail.com \\ Received 21 August, 2009; Revised 29 March, 2010
}

\begin{abstract}
In this paper, we establish a fixed point theorem for a pair of self maps satisfying a general contractive condition of integral type. This theorem extends and generalizes some early results of Boikanyo[2].
\end{abstract}

2000 AMS Subject Classification: 54H25, 47H10.

Key Words: Lebesgue-integrable map, Complete metric space, Common fixed point.

\section{INTRODUCTION}

The first well known result on fixed points for contractive map was Banach - Cacciopoli theorem, published in 1922 (see [1], [4]). In general setting of complete metric space, Smart ([11]) presented the following result.

Theorem 1.1. Let $(X, d)$ be a complete metric space, $c \in[0,1)$, and let $T: X \rightarrow X$ be a map such that for each $x, y \in X$,

$$
d(T x, T y) \leq c d(x, y)
$$

Then, $T$ has a unique fixed point $z \in X$ such that for each $x \in X, \lim _{n \rightarrow \infty} T^{n} x=z$.

After this classical result, many theorems dealing with maps satisfying various types of contractive inequalities have been established (see for details [2], [5]-[10], [12]).

In 2002, Branciari ([3]) obtained the following theorem.

Theorem 1.2. Let $(X, d)$ be a complete metric space, $c \in[0,1)$, and let $T: X \rightarrow X$ be a map such that for each $x, y \in X$,

$$
\int_{0}^{d(T x, T y)} \varphi(t) d t \leq c \int_{0}^{d(x, y)} \varphi(t) d t
$$


where $\varphi: R^{+} \rightarrow R^{+}$is a Lebesgue - integrable map which is summable, non negative and such that $\int_{0}^{\varepsilon} \varphi(t) d t>0$ for each $\varepsilon>0$. Then, $T$ has a unique fixed point $z \in X$ and for each $x \in X$, $\lim _{n \rightarrow \infty} T^{n} x=z$.

In 2007, Boikanyo [2] proved some fixed point theorems for a self map satisfying a general contractive condition of integral type as an extension of Branciari's theorem. In [3], it was mentioned that one can generalize other results related to contractive conditions of some kind, such as in [8].

The main purpose of our paper is to obtain some results for a pair of self maps satisfying a general contractive condition of integral type.

Throughout this paper, $N$ denotes the set of natural numbers.

\section{Main Results}

Theorem 2.1. Let $(X, d)$ be a complete metric space. Let $a_{i}(i=1,2,3,4,5)$ be non negative real

numbers satisfying $\sum_{i=1}^{5} a_{i}<1, T_{1}$ and $T_{2}$ be a pair of self maps of the metric space $X$ such that for each $x, y \in X$

$$
\begin{aligned}
\int_{0}^{d\left(T_{1} x, T_{2} y\right)} \varphi(t) d t \leq a_{1} \int_{0}^{d(x, y)} \varphi(t) d t+a_{2} \int_{0}^{d\left(x, T_{1} x\right)} \varphi(t) d t & \\
& \quad+a_{3} \int_{0}^{d\left(y, T_{2} y\right)} \varphi(t) d t+a_{4} \int_{0}^{d\left(x, T_{2} y\right)} \varphi(t) d t+a_{5} \int_{0}^{d\left(y, T_{1} x\right)} \varphi(t) d t
\end{aligned}
$$

where $\varphi: R^{+} \rightarrow R^{+}$is a Lebesgue-integrable map which is summable, non-negative and such that $\int_{0}^{\varepsilon} \varphi(t) d t>0$ for each $\varepsilon>0$. Then $T_{1}$ and $T_{2}$ have a unique common fixed point $z \in X$.

Proof. Let $x_{0}$ be any point of $X$.

$$
\text { Define } \begin{aligned}
x_{2 n-1} & =T_{1} x_{2 n-2} \\
x_{2 n} & =T_{2} x_{2 n-1} \quad \text { where } n \in N
\end{aligned}
$$

We claim that $\quad \lim _{n \rightarrow \infty} d\left(x_{n}, x_{n+1}\right)=0$.

To prove (2.2), we require to show that

$$
\int_{0}^{d\left(x_{n}, x_{n+1}\right)} \varphi(t) d t \leq r^{n} \int_{0}^{d\left(x_{0}, x_{1}\right)} \varphi(t) d t \text { where } r=\frac{2 a_{1}+a_{2}+a_{3}+a_{4}+a_{5}}{2-a_{2}-a_{3}-a_{4}-a_{5}}<1
$$


For this, by interchanging $x$ with $y$ and $T_{1}$ with $T_{2}$ in (2.1), we obtain

$$
\begin{aligned}
\int_{0}^{d\left(T_{2} y, T_{1} x\right)} \varphi(t) d t \leq & a_{1} \int_{0}^{d(y, x)} \varphi(t) d t+a_{2} \int_{0}^{d\left(y, T_{2} y\right)} \varphi(t) d t \\
& +a_{3} \int_{0}^{d\left(x, T_{1} x\right)} \varphi(t) d t+a_{4} \int_{0}^{d\left(y, T_{1} x\right)} \varphi(t) d t+a_{5} \int_{0}^{d\left(x, T_{2} y\right)} \varphi(t) d t
\end{aligned}
$$

Now, from (2.1), (2.3) and using symmetric property, we obtain

$$
\int_{0}^{d\left(T_{1} x, T_{2} y\right)} \varphi(t) d t \leq a_{1} \int_{0}^{d(x, y)} \varphi(t) d t+\left(\frac{a_{2}+a_{3}}{2}\right) \int_{0}^{d\left(x, T_{1} x\right)} \varphi(t) d t+\left(\frac{a_{2}+a_{3}}{2}\right) \int_{0}^{d\left(y, T_{2} y\right)} \varphi(t) d t
$$

$+\left(\frac{a_{4}+a_{5}}{2}\right) \int_{0}^{d\left(x, T_{2} y\right)} \varphi(t) d t+\left(\frac{a_{4}+a_{5}}{2}\right) \int_{0}^{d\left(y, T_{1} x\right)} \varphi(t) d t$

Using (2.4) for odd $n$, we obtain

$$
\begin{aligned}
\int_{0}^{d\left(x_{n}, x_{n+1}\right)} \varphi(t) d t & =\int_{0}^{d\left(T_{1} x_{n-1}, T_{2} x_{n}\right)} \varphi(t) d t \\
\leq & a_{1} \int_{0}^{d\left(x_{n-1}, x_{n}\right)} \varphi(t) d t+\left(\frac{a_{2}+a_{3}}{2}\right) \int_{0}^{d\left(x_{n-1}, T_{1} x_{n-1}\right)} \varphi(t) d t+\left(\frac{a_{2}+a_{3}}{2}\right) \int_{0}^{d\left(x_{n}, T_{2} x_{n}\right)} \varphi(t) d t \\
& +\left(\frac{a_{4}+a_{5}}{2}\right) \int_{0}^{d\left(x_{n-1}, T_{2} x_{n}\right)} \varphi(t) d t+\left(\frac{a_{4}+a_{5}}{2}\right) \int_{0}^{d\left(x_{n}, T_{1} x_{n-1}\right)} \varphi(t) d t \\
= & a_{1} \int_{0}^{d\left(x_{n-1}, x_{n}\right)} \varphi(t) d t+\left(\frac{a_{2}+a_{3}}{2}\right) \int_{0}^{d\left(x_{n-1}, x_{n}\right)} \varphi(t) d t+\left(\frac{a_{2}+a_{3}}{2}\right) \int_{0}^{d\left(x_{n}, x_{n+1}\right)} \varphi(t) d t \\
& +\left(\frac{a_{4}+a_{5}}{2}\right) \int_{0}^{d\left(x_{n-1}, x_{n+1}\right)} \varphi(t) d t+\left(\frac{a_{4}+a_{5}}{2}\right) \int_{0}^{d\left(x_{n}, x_{n}\right)} \varphi(t) d t
\end{aligned}
$$

Again, using (2.4) for even $n$, we obtain

$$
\begin{aligned}
\int_{0}^{d\left(x_{n}, x_{n+1}\right)} \varphi(t) d t & =\int_{0}^{d\left(T_{2} x_{n-1}, T_{1} x_{n}\right)} \varphi(t) d t \\
\leq & a_{1} \int_{0}^{d\left(x_{n-1}, x_{n}\right)} \varphi(t) d t+\left(\frac{a_{2}+a_{3}}{2}\right) \int_{0}^{d\left(x_{n-1}, T_{2}, x_{n-1}\right)} \varphi(t) d t+\left(\frac{a_{2}+a_{3}}{2}\right) \int_{0}^{d\left(x_{n}, T_{1} x_{n}\right)} \varphi(t) d t \\
& +\left(\frac{a_{4}+a_{5}}{2}\right) \int_{0}^{d\left(x_{n-1}, T_{1} x_{n}\right)} \varphi(t) d t+\left(\frac{a_{4}+a_{5}}{2}\right) \int_{0}^{d\left(x_{n}, T_{2} x_{n-1}\right)} \varphi(t) d t \\
= & a_{1} \int_{0}^{d\left(x_{n-1}, x_{n}\right)} \varphi(t) d t+\left(\frac{a_{2}+a_{3}}{2}\right) \int_{0}^{d\left(x_{n-1}, x_{n}\right)} \varphi(t) d t+\left(\frac{a_{2}+a_{3}}{2}\right) \int_{0}^{d\left(x_{n}, x_{n+1}\right)} \varphi(t) d t
\end{aligned}
$$




$$
+\left(\frac{a_{4}+a_{5}}{2}\right) \int_{0}^{d\left(x_{n-1}, x_{n+1}\right)} \varphi(t) d t+\left(\frac{a_{4}+a_{5}}{2}\right) \int_{0}^{d\left(x_{n}, x_{n}\right)} \varphi(t) d t
$$

From the above two cases, one can see that

$$
\begin{aligned}
\int_{0}^{d\left(x_{n}, x_{n+1}\right)} \varphi(t) d t \leq & a_{1} \int_{0}^{d\left(x_{n-1}, x_{n}\right)} \varphi(t) d t+\left(\frac{a_{2}+a_{3}}{2}\right) \int_{0}^{d\left(x_{n-1}, x_{n}\right)} \varphi(t) d t+\left(\frac{a_{2}+a_{3}}{2}\right) \int_{0}^{d\left(x_{n}, x_{n+1}\right)} \varphi(t) d t \\
& +\left(\frac{a_{4}+a_{5}}{2}\right) \int_{0}^{d\left(x_{n-1}, x_{n+1}\right)} \varphi(t) d t+\left(\frac{a_{4}+a_{5}}{2}\right) \int_{0}^{d\left(x_{n}, x_{n}\right)} \varphi(t) d t \\
\leq & a_{1} \int_{0}^{d\left(x_{n-1}, x_{n}\right)} \varphi(t) d t+\left(\frac{a_{2}+a_{3}}{2}\right) \int_{0}^{d\left(x_{n-1}, x_{n}\right)} \varphi(t) d t+\left(\frac{a_{2}+a_{3}}{2}\right) \int_{0}^{d\left(x_{n}, x_{n+1}\right)} \varphi(t) d t \\
& +\left(\frac{a_{4}+a_{5}}{2}\right) \int_{0}^{d\left(x_{n-1}, x_{n}\right)} \varphi(t) d t+\left(\frac{a_{4}+a_{5}}{2}\right) \int_{0}^{d\left(x_{n}, x_{n+1}\right)} \varphi(t) d t
\end{aligned}
$$

It follows that $\int_{0}^{d\left(x_{n}, x_{n+1}\right)} \varphi(t) d t \leq\left(\frac{2 a_{1}+a_{2}+a_{3}+a_{4}+a_{5}}{2-a_{2}-a_{3}-a_{4}-a_{5}}\right) \int_{0}^{d\left(x_{n-1}, x_{n}\right)} \varphi(t) d t$

$$
\begin{aligned}
& =r \int_{0}^{d\left(x_{n-1}, x_{n}\right)} \varphi(t) d t \\
& \leq r^{n} \int_{0}^{d\left(x_{0}, x_{1}\right)} \varphi(t) d t \rightarrow 0 \text { as } n \rightarrow \infty \text { since } r<1 \text {, owing to the }
\end{aligned}
$$

assumption $\sum_{i=1}^{5} a_{i}<1$. Therefore, $\lim _{n \rightarrow \infty} d\left(x_{n}, x_{n+1}\right)=0$.

Now, we show that $\left\{x_{n}\right\}$ is a Cauchy sequence in $X$. Let $m>n$ where $m, n \in N$

Without any loss of generality, two cases arise:

(i) $m$ is even when $n$ is odd

and (ii) $m$ is odd when $n$ is even.

Case I: We choose $n$ and $m$ to be odd and even respectively

Then we have

$$
\begin{aligned}
\int_{0}^{d\left(x_{n}, x_{m}\right)} \varphi(t) d t= & \int_{0}^{d\left(T_{1} x_{n-1}, T_{2} x_{m-1}\right)} \varphi(t) d t \\
\leq & a_{1} \int_{0}^{d\left(x_{n-1}, x_{m-1}\right)} \varphi(t) d t+a_{2} \int_{0}^{d\left(x_{n-1}, T_{1} x_{n-1}\right)} \varphi(t) d t+a_{3} \int_{0}^{d\left(x_{m-1}, T_{2} x_{m-1}\right)} \varphi(t) d t \\
& +a_{4} \int_{0}^{d\left(x_{n-1}, T_{2} x_{m-1}\right)} \varphi(t) d t+a_{5} \int_{0}^{d\left(x_{m-1}, T_{1} x_{n-1}\right)} \varphi(t) d t \\
= & a_{1} \int_{0}^{d\left(x_{n-1}, x_{m-1}\right)} \varphi(t) d t+a_{2} \int_{0}^{d\left(x_{n-1}, x_{n}\right)} \varphi(t) d t+a_{3} \int_{0}^{d\left(x_{m-1}, x_{m}\right)} \varphi(t) d t
\end{aligned}
$$




$$
+a_{4} \int_{0}^{d\left(x_{n-1}, x_{m}\right)} \varphi(t) d t+a_{5} \int_{0}^{d\left(x_{m-1}, x_{n}\right)} \varphi(t) d t
$$

Case II: We choose $n$ and $m$ to be even and odd respectively.

Then we have

$$
\begin{aligned}
\int_{0}^{d\left(x_{n}, x_{m}\right)} \varphi(t) d t= & \int_{0}^{d\left(T_{2} x_{n-1}, T_{1} x_{m-1}\right)} \varphi(t) d t \\
\leq & a_{1} \int_{0}^{d\left(x_{n-1}, x_{m-1}\right)} \varphi(t) d t+a_{2} \int_{0}^{d\left(x_{n-1}, T_{2} x_{n-1}\right)} \varphi(t) d t+a_{3} \int_{0}^{d\left(x_{m-1}, T_{1} x_{m-1}\right)} \varphi(t) d t \\
& +a_{4} \int_{0}^{d\left(x_{n-1}, T_{1} x_{m-1}\right)} \varphi(t) d t+a_{5} \int_{0}^{d\left(x_{m-1}, T_{2} x_{n-1}\right)} \varphi(t) d t \\
= & a_{1} \int_{0}^{d\left(x_{n-1}, x_{m-1}\right)} \varphi(t) d t+a_{2} \int_{0}^{d\left(x_{n-1}, x_{n}\right)} \varphi(t) d t+a_{3} \int_{0}^{d\left(x_{m-1}, x_{m}\right)} \varphi(t) d t \\
& +a_{4} \int_{0}^{d\left(x_{n-1}, x_{m}\right)} \varphi(t) d t+a_{5} \int_{0}^{d\left(x_{m-1}, x_{n}\right)} \varphi(t) d t
\end{aligned}
$$

From both the cases, we have

$$
\begin{aligned}
\int_{0}^{d\left(x_{n}, x_{m}\right)} \varphi(t) d t \leq & a_{1} \int_{0}^{d\left(x_{n-1}, x_{m-1}\right)} \varphi(t) d t+a_{2} \int_{0}^{d\left(x_{n-1}, x_{n}\right)} \varphi(t) d t+a_{3} \int_{0}^{d\left(x_{m-1}, x_{m}\right)} \varphi(t) d t \\
& +a_{4} \int_{0}^{d\left(x_{n-1}, x_{m}\right)} \varphi(t) d t+a_{5} \int_{0}^{d\left(x_{m-1}, x_{n}\right)} \varphi(t) d t \\
\leq & a_{1} \int_{0}^{d\left(x_{n-1}, x_{n}\right)} \varphi(t) d t+a_{1} \int_{0}^{d\left(x_{n}, x_{m}\right)} \varphi(t) d t+a_{1} \int_{0}^{d\left(x_{m}, x_{m-1}\right)} \varphi(t) d t \\
& +a_{2} \int_{0}^{d\left(x_{n-1}, x_{n}\right)} \varphi(t) d t+a_{3} \int_{0}^{d\left(x_{m-1}, x_{m}\right)} \varphi(t) d t+a_{4} \int_{0}^{d\left(x_{n}, x_{m}\right)} \varphi(t) d t \\
& +a_{4} \int_{0}^{d\left(x_{n-1}, x_{n}\right)} \varphi(t) d t+a_{5} \int_{0}^{d\left(x_{m-1}, x_{m}\right)} \varphi(t) d t+a_{5} \int_{0}^{d\left(x_{m}, x_{n}\right)} \varphi(t) d t
\end{aligned}
$$

Therefore

$$
\begin{gathered}
\int_{0}^{d\left(x_{n}, x_{m}\right)} \varphi(t) d t \leq\left(\frac{a_{1}+a_{2}+a_{4}}{1-a_{1}-a_{4}-a_{5}}\right) \int_{0}^{d\left(x_{n-1}, x_{n}\right)} \varphi(t) d t+\left(\frac{a_{1}+a_{3}+a_{5}}{1-a_{1}-a_{4}-a_{5}}\right) \int_{0}^{d\left(x_{m-1}, x_{m}\right)} \varphi(t) d t \\
\leq\left(\frac{a_{1}+a_{2}+a_{4}}{1-a_{1}-a_{4}-a_{5}}\right) r^{n-1} \int_{0}^{d\left(x_{0}, x_{1}\right)} \varphi(t) d t+\left(\frac{a_{1}+a_{3}+a_{5}}{1-a_{1}-a_{4}-a_{5}}\right) r^{m-1} \int_{0}^{d\left(x_{0}, x_{1}\right)} \varphi(t) d t \\
\rightarrow 0 \text { as } n, m \rightarrow \infty, \text { since } r<1
\end{gathered}
$$

Hence, $\left\{x_{n}\right\}$ is a Cauchy sequence in the complete metric space $X$, so it is convergent in $X$. 
Let its limit be $z$, i.e. $\lim _{n \rightarrow \infty} x_{n}=z$. We show that $T_{1} z=T_{2} z=z$.

Now, we have

$$
\begin{aligned}
& \int_{0}^{d\left(x_{2 n}, T_{1} z\right)} \varphi(t) d t=\int_{0}^{d\left(T_{2} x_{2 n-1}, T_{1} z\right)} \varphi(t) d t \\
& \leq a_{1} \int_{0}^{d\left(x_{2 n-1}, z\right)} \varphi(t) d t+a_{2} \int_{0}^{d\left(x_{2 n-1}, T_{2} x_{2 n-1}\right)} \varphi(t) d t+a_{3} \int_{0}^{d\left(z, T_{1} z\right)} \varphi(t) d t \\
& \quad+a_{4} \int_{0}^{d\left(x_{2 n-1}, T_{1} z\right)} \varphi(t) d t+a_{5} \int_{0}^{d\left(z, T_{2} x_{2 n-1}\right)} \varphi(t) d t \\
& =a_{1} \int_{0}^{d\left(x_{2 n-1}, z\right)} \varphi(t) d t+a_{2} \int_{0}^{d\left(x_{2 n-1}, x_{2 n}\right)} \varphi(t) d t+a_{3} \int_{0}^{d\left(z, T_{1} z\right)} \varphi(t) d t \\
& \quad+a_{4} \int_{0}^{d\left(x_{2 n-1}, T_{1} z\right)} \varphi(t) d t+a_{5} \int_{0}^{d\left(z, x_{2 n}\right)} \varphi(t) d t
\end{aligned}
$$

Taking the limit as $n \rightarrow \infty$, we get

$$
\begin{aligned}
& \int_{0}^{d\left(z, T_{1} z\right)} \varphi(t) d t \leq a_{3} \int_{0}^{d\left(z, T_{1} z\right)} \varphi(t) d t+a_{4} \int_{0}^{d\left(z, T_{1} z\right)} \varphi(t) d t \\
& \Rightarrow \int_{0}^{d\left(z, T_{1} z\right)} \varphi(t) d t=0 \\
& \Rightarrow z=T_{1} z
\end{aligned}
$$

Similarly, it may be shown that $T_{2} z=z$. Thus $T_{1}$ and $T_{2}$ have a common fixed point.

For uniqueness, if possible, let $w$ be another common fixed point of $T_{1}$ and $T_{2}$ such that $w \neq z$.

Now, we have

$$
\begin{aligned}
\int_{0}^{d(z, w)} \varphi(t) d t= & \int_{0}^{d\left(T_{1} z, T_{2} w\right)} \varphi(t) d t \\
\leq & a_{1} \int_{0}^{d(z, w)} \varphi(t) d t+a_{2} \int_{0}^{d\left(z, T_{1} z\right)} \varphi(t) d t+a_{3} \int_{0}^{d\left(w, T_{2} w\right)} \varphi(t) d t \\
& +a_{4} \int_{0}^{d\left(z, T_{2} w\right)} \varphi(t) d t+a_{5} \int_{0}^{d\left(w, T_{1} z\right)} \varphi(t) d t \\
= & a_{1} \int_{0}^{d(z, w)} \varphi(t) d t+a_{4} \int_{0}^{d(z, w)} \varphi(t) d t+a_{5} \int_{0}^{d(w, z)} \varphi(t) d t \\
\Rightarrow \int_{0}^{d(z, w)} \varphi(t) d t & =0, \text { a contradiction. Hence, } z=w .
\end{aligned}
$$

Thus $T_{1}$ and $T_{2}$ have a unique common fixed point. This completes the proof. 
Corollary 2.2. Let $(X, d)$ be a complete metric space. Let $a, b, c$ be non negative real numbers satisfying $a+b+c<1, T_{1}$ and $T_{2}$ be a pair of self maps of the metric space $X$ into itself such that for each $x, y \in X$,

$$
\int_{0}^{d\left(T_{1} x, T_{2} y\right)} \varphi(t) d t \leq a \int_{0}^{d\left(x, T_{1} x\right)} \varphi(t) d t+b \int_{0}^{d\left(y, T_{2} y\right)} \varphi(t) d t+c \int_{0}^{d(x, y)} \varphi(t) d t
$$

where $\varphi: R^{+} \rightarrow R^{+}$is a Lebesgue-integrable map which is summable, non-negative and such that $\int_{0}^{\varepsilon} \varphi(t) d t>0$ for each $\varepsilon>0$. Then $T_{1}$ and $T_{2}$ have a unique common fixed point $z \in X$.

Proof. Since the contractive condition (2.5) is obviously a special case of (2.1) by setting $a_{1}=c, a_{2}=a, a_{3}=b$ and $a_{4}=a_{5}=0$, the result follows immediately from Theorem 2.1. $\square$

Corollary 2.3. Let $(X, d)$ be a complete metric space. Let $a, b, c$ be non negative real numbers satisfying $a+b+c<1, T_{1}$ and $T_{2}$ be a pair of self maps of the metric space $X$ such that for each $x, y \in X$,

$$
\int_{0}^{d\left(T_{1} x, T_{2} y\right)} \varphi(t) d t \leq a \int_{0}^{d\left(x, T_{2} y\right)} \varphi(t) d t+b \int_{0}^{d\left(y, T_{1} x\right)} \varphi(t) d t+c \int_{0}^{d(x, y)} \varphi(t) d t
$$

where $\varphi: R^{+} \rightarrow R^{+}$is a Lebesgue-integrable map which is summable, non-negative and such that $\int_{0}^{\varepsilon} \varphi(t) d t>0$ for each $\varepsilon>0$. Then $T_{1}$ and $T_{2}$ have a unique common fixed point $z \in Z$.

Proof. Since the contractive condition (2.6) is also a special case of (2.1) by letting $a_{1}=c$, $a_{4}=a, a_{5}=b$ and $a_{2}=a_{3}=0$, the result follows immediately from Theorem 2.1.

Remark 2.4. We give some remarks which clarify the connection between our results and the results obtained in [2].

(i) Theorem 1 and 2(cf. [2]) are special cases of Corollary 2.2 and 2.3 respectively with $T_{1}=T_{2}$, $a=b$ and $c=0$.

(ii) By taking $T_{1}=T_{2}$, Corollary 2.2 and 2.3 reduce Theorem 3 and 4 (cf. [2]) respectively.

(iii) Theorem 5 (cf. [2]) is a consequence of Theorem 2.1 if we take $T_{1}=T_{2}$. 


\section{REFERENCES}

1. S. Banach, 1922. Sur les operations dans les ensembles abstraits et leur application aux equations integrales, Fund. Math. 3: 133 - 181 ( in French )

2. O. A. Boikanyo, 2007. Some fixed point theorems for mappings satisfying a general contractive condition of integral types, Far East J. Math. Sci., (FJMS) 26 (1)219 - 230.

3. A. Branciari, 2002. A fixed point theorem for mappings satisfying a general contractive condition of integral type, Int. J. Math. Sci. 29: 531 - 536.

4. R. Caccioppoli, 1930. Un teorema generale sull' esistenza di elementi uniti in una transformazione funzionale, Rend. Acad. Dei Lincei 11: 794 - 799 ( in Italian).

5. S.K. Chatterjea, 1972. Fixed-point theorems, C.R. Acad. Balgare Sci. 25: 727 - 730.

6. G. E. Hardy and T. D. Rogers, 1973. A generalization of a fixed point theorem of Reich, Bull.Cand. Math. Soc. 16:201 -206.

7. R. Kannan, Some results on fixed points, Bull. Calcutta Math. Soc. 60 (1968), 71 - 76.

8. B. E. Rhoades, A comparison of various definitions of contractive mappings, Trans. Amer. Math. Soc. 226 (1977), 257 - 290.

9. B. E. Rhoades, Two fixed point theorems for mappings satisfying a general contractive condition of integral type, Int. J. Math. Math. Sci. 63 (2003), 4007 - 4013.

10. M. Sen Gupta ( Mrs. Das Gupta ), On common fixed points of operators, Bull. Cal. Math. Soc. 66 (1974) $149-153$.

11. D. R. Smart, Fixed point theorems, Cambridge University Press, London, 1974.

12. P. Vijayaraju, B.E. Rhoades and R. Mohanraj, A fixed point theorem for a pair of maps satisfying a general contractive condition of integral type, Int. J. Math. Math. Sci. 15 (2005), $2359-2364$. 